\title{
Mercury intoxication presenting with hypertension and tachycardia
}

\author{
Willi Wößmann, Martina Kohl, Gunnar Grüning, Peter Bucsky
}

\begin{abstract}
An 11 year old girl presented with hypertension and tachycardia. Excess urinary catecholamine excretion suggested phaeochromocytoma but imaging studies failed to demonstrate a tumour. Other symptoms included insomnia and weight loss, and she was found to have a raised concentration of mercury in blood and urine. Mercury intoxication should be considered in the differential diagnosis of hypertension with tachycardia even in patients presenting without the skin lesions typical of mercury intoxication and without a history of exposure. (Arch Dis Child 1999;80:556-557)
\end{abstract}

Keywords: hypertension; tachycardia; mercury poisoning; phaeochromocytoma

The differential diagnosis of hypertension, tachycardia, weight loss, and psychiatric symptoms includes thyrotoxicosis, drugs - for example, cocaine, and catecholamine excess. The latter can be caused by a catecholamine producing tumour - usually a phaeochromocytoma - or by mercury intoxication. A careful history and clinical examination usually provides sufficient evidence to distinguish between acrodynia and phaeochromocytoma. Acrodynia may be an appropriate diagnosis if a toddler shows characteristic skin manifestations and there is an obvious source of intoxication. This can be confirmed by the demonstration of increased mercury concentrations in blood and urine. ${ }^{1-3}$

We report a case of an 11 year old girl with mercury intoxication who presented with hypertension, tachycardia and catecholamine

Accepted 19 January 1999

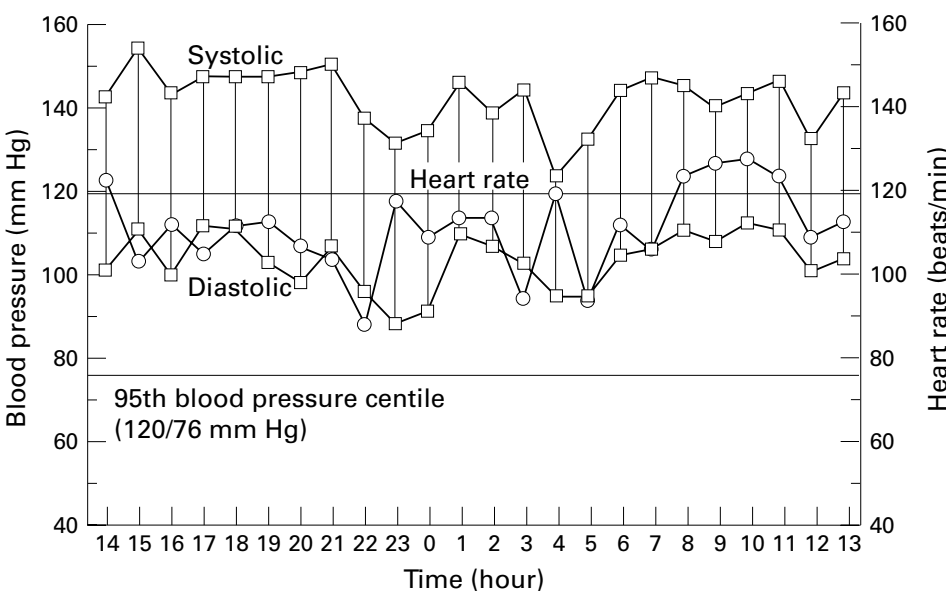

Figure 1 Mean hourly systolic and diastolic blood pressure and heart rate of the patient at presentation by oscillometric 24 hour blood pressure monitoring. excess but without cutaneous lesions or a history of exposure to mercury.

\section{Case report}

A Taiwanese girl was admitted to our institution because of hypertension (160/120 $\mathrm{mm} \mathrm{Hg}$ ) and tachycardia (120 beats/min). She had suffered from painful itching in her extremities but this had resolved before admission. Oscillometric 24 hour blood pressure monitoring revealed severe hypertension without nocturnal dipping (fig 1). Exposure to drugs and toxins was denied, and there was no family history of hypertension or malignant disease. Laboratory values showed normal thyroid and kidney function. Urinary concentrations of vanillylmandelic acid (VMA) were slightly raised (5.6-5.8 $\mathrm{nmol} / \mu \mathrm{mol}$ creatinine; normal < 4.7); homovanillic acid (HMA) was within the normal range. Investigations including abdominal and cervical ultrasound, computed tomography scans, and an $\mathrm{M}$-iodobenzylguanidine scan did not reveal a tumour. She was treated with enalapril. After failing to attend for follow up, she presented two months later with insomnia, depression, daytime fatigue, and loss of $12 \mathrm{~kg}$ body weight (initial body weight was $36 \mathrm{~kg}$ ). On examination, she could barely stand because of weakness and ataxia. She was irritable and remained hypertensive despite medication. Nerve conduction velocity of the peroneal and median nerves was reduced and a cerebral magnetic resonance imaging scan showed no abnormality. Again, catecholamine concentrations (noradrenaline (norepinephrine), dopamine, and VMA were slightly raised. A screen for heavy metals was performed despite no history of exposure. The blood and urine of the patient (but not of her parents and younger sister) showed very high mercury concentrations (blood $20 \mu \mathrm{g} / \mathrm{l}$, normal for adults < 10; urine $217 \mu \mathrm{g} / 1$, normal for adults $<20$ ). Direct questioning did not reveal any obvious sources of mercury intoxication such as recreational use, presence of a broken thermometer or use of old ointments or Chinese cups during the last year. During treatment with D-penicillamine, mercury concentrations returned to normal. At four month's follow up her heart rate and blood pressure (without medication) were normal. She gained weight, and ataxia resolved slowly over one year.

\section{Discussion}

A catecholamine producing tumour is not the only condition that causes hypertension and tachycardia as well as increased catecholamine concentrations. In inorganic mercury poisoning, the metal combines with the sulphydryl 
group of S-adenosylmethionine, which acts as a cofactor for catecholamine-Omethyltransferase (COMT). COMT inhibition leads to accumulation of catecholamines, typically noradrenaline, adrenaline (epinephrine), and dopamine, and to a lesser degree VMA, but not usually HVA. ${ }^{24}$ The sympathetic overactivity explains the haemodynamic symptoms of acrodynia. Curiously, in our patient, VMA was the catecholamine most predominantly excreted. It is usual for mercury intoxication to show a much lesser degree of catecholamine excess than would normally be expected in a phaeochromocytoma or neuroblastoma.

Clinical signs of the fully developed form of acrodynia include pronounced mental changes such as insomnia and irritability, pain in the extremities, typical skin lesions (hence the nickname "pink disease"), profuse sweating, and anorexia as well as hypertension and tachycardia. ${ }^{5}$ Uncharacteristically, our patient did not have even a transitory rash and was not sweating excessively. However, desquamation and pink palms and soles are unusual in children beyond toddler age because of increased skin thickness.

Since mercury has been excluded from teething powders and ointments, inhalation of mercury from broken thermometers has accounted for most described poisonings in toddlers, ${ }^{1-3}$ and recreational use and inorganic mercury warming have been suggested as further causes of intoxication. ${ }^{4}$ In this case, no source could be found even after repeated questioning of family members. We considered exposure at home to be unlikely as the family of our patient live together in a small apartment. Her parents and sister excreted only traces of the heavy metal and we did not test the mercury levels in her home. A Chinese medicine containing a metal substance had been given to the girl, but was found to contain only traces of mercury. The child was, therefore, placed under constant surveillance. Repeated psychological evaluation of both parents and children did not indicate child abuse, but this cannot be excluded.

It is not known how many transient unexplained hypertensive episodes in children might be caused by mercury intoxication. Even though acrodynia is rare, its diagnosis can be made by a single urine analysis and, therefore, acrodynia should be excluded before expensive and invasive procedures are performed to discount phaeochromocytoma as the cause of the symptoms.

1 Velzeboer SCJM, Frenkel J, de Wolff FA. A hypertensive toddler. Lancet 1997;349:1810.

2 Baudouin V, Bocquet N, Rybojad M, et al. Clinical quiz. Pediatr Nephrol 1997;11:263-4.

3 Von Muehlendahl KE. Intoxication from mercury spilt on carpets. Lancet 1990;336:1578.

4 Henningsson C, Hoffmann S, McGonigle L, Winter JSD. Acute mercury poisoning (acrodynia) mimicking phaeo-

5 Warkany J. Acrodynia-postmortem of a disease. Am $\mathcal{F} \mathrm{Dis}$ Child 1966;112:147-56. 